\title{
GINGIVAL MICROLEAKAGE OF DENTAL AMALGAM, BULK FILL COMPOSITE RESIN AND ALKASITE BASED COMPOSITE RESTORATIONS IN CLASS II CAVITIES
}

\author{
Asmaa Mohamed Abdallah*
}

\begin{abstract}
Objectives: To evaluate and compare gingival microleakage in class II cavities restored with amalgam, bulk-fill composite, and the new alkasite based dental composite (Cention-N).

Materials and Methods: Two independant Class II cavities were prepared in forty extracted human third molars. Each molar had a mesial cavity with gingival margin located $1 \mathrm{~mm}$ occlusal to the cemento-enamel junction (supra CEJ) and a distal cavity with gingival margin located $1 \mathrm{~mm}$ apical to the CEJ (sub CEJ). The prepared molars were randomly assigned to four groups $(n=10)$ according to the restorative material used. Group 1; was restored with dental amalgam. Group 2; was restored with bulk fill dental composite. Group 3; was restored with alkasite based composite without bonding agent. Group 4; was restored with alkasite based composite with bonding agent. The teeth were then thermocycled and immersed in methylene blue solution for $24 \mathrm{~h}$. The teeth were mesiodistally sectioned and evaluated under a stereomicroscope at $\times 10$ magnification. Dye penetration scorings were recorded, and data were analyzed Kruskal Wallis followed by post-hoc Dunn's test.
\end{abstract}

Results: Dental amalgam showed the least microleakage and came to be better than the Cention-N and bulkfill dental composite. Microleakage for Cention-N without bonding agent was the highest of all. In terms of supra CEJ and sub CEJ microleakage; the difference for group 1and 3 was nonsignificant but was significant for group 2 and 4 .

Conclusions: Alkasite based dental composite with bonding agent in class II restorations are not significantly different from Bulk fill composites in terms of microleakage

KEYWORDS: Alkasite based composite, bulk fill Composite Resins, Dental amalgam, Microeakage, Class II

* Assistant Professor Operative Dentistry Department, Faculty of Dentistry, Mansoura University. 


\section{INTRODUCTION}

Microleakage is the percolation of bacteria, liquids, molecules and ions via the cavity wall/restoration interface ${ }^{1}$ leading to pulpal pathology. So, decreasing microleakage is an important goal in operative dentistry ${ }^{2}$. A uniform sealed interface is important to enhance the durability of restoration ${ }^{3}$ which is challenging especially in class II restorations with gingival margin extending below the gingival tissues ${ }^{4}$. Obtaining sealed Gingival margin is difficult due to its position near the gingival crevice which makes cavity preparation, moisture control, and bonding procedure difficult. Dentin margins contain less inorganic content than enamel, contain more moisture and show more leakage ${ }^{5}$.

Amalgam has long track record of success as direct restorative material in posterior teeth; due to low cost, long-term clinical evaluation, good wear resistance, self-sealing ability, low techniquesensitivity, easy to place in a single appointment and non-abrasive to opposing teeth in function. Unfortunately; metallic color of dental amalgam, lack of bonding to tooth structure, leading to unnecessary removal of sound tooth structure to provide retention, and concerns about mercury toxicity decreased the popularity of amalgam. ${ }^{6}$

Direct composite restorations have gained popularity over amalgam restorations because of their superior esthetic properties, micromechanical retention, and no mercury content. However, polymerization shrinkage remains a major drawback. ${ }^{7}$ Polymerization shrinkage stresses cause micro-cracks in composite, bond failure at the restoration/tooth interface and resultant formation of micro-gaps, marginal microleakage and postoperative hypersensitivity $^{8,9}$. Decreasing the polymerization shrinkage stresses of composites is mandatory to enhance marginal adaptation and the longevity of composite restorations ${ }^{10,11}$.

Polymerization shrinkage can be reduced by layering application of composite resins (to decrease the $\mathrm{C}$ factor), use of different polymerization techniques, using a base under the restoration, and increasing the filler content ${ }^{12,13}$. The drawbacks of incremental application of composite resins such as, voids and contamination, bond failure between layers, and time-consuming nature ${ }^{14}$, resulted in the introduction of bulk fill composites having more filler content, larger filler size and higher translucency than conventional composites ${ }^{15}$. Bulk application technique is simpler as it makes the treatment quicker by decreasing clinical steps thus making it minimally technique sensitive. Due to having different monomers and higher filler contents bulk fill composites reduced shrinkage stress ${ }^{16}$. They can be used in $4 \mathrm{~mm}$ thick increments without affecting their mechanical properties or conversion degree $^{17,18}$. Less polymerization shrinkage ${ }^{19}$, lower cuspal flexure in standard class II cavities ${ }^{20}$, acceptable bond strength regardless of the cavity form and technique of filling ${ }^{21}$.

Dentists have long sought a material which can combine the strength of amalgam and the esthetics of dental composite. The recently introduced material alkasite based composite (Cention- $\mathrm{N}$ ) have been developed as an esthetic amalgam alternative, with comparable compressive strength. It is a resin based, powder-liquid restorative material which can be used in self-curing mode or light curing mode. According to the manufacturer, $\mathrm{CN}$ contains three inorganic glasses: barium alumino-silicate glass, calcium barium alumino-fluoro-silicate and a basic calcium fluoro-silicate glass referred to as an "Alkasite"filler ${ }^{22}$.

This in vitro study aimed to evaluate and compare the gingival microleakage in Class II cavities (supra CEJ and sub CEJ) restored with amalgam, bulk-fill composite and the newly introduced material Cention-N. The null hypothesis of the study was that there is no significant difference in microleakage of the evaluated materials. 


\section{MATERIALS AND METHODS}

\section{Sample preparation}

Forty extracted human third molars, free of cracks, caries and restorations were used in this study. The specimens were cleaned with pumice and stored in $0.5 \%$ chloramine $\mathrm{T}$ at $4^{\circ} \mathrm{C}$ for 2 weeks. 80 Class II cavities were prepared using a cylindrical round end diamond bur (838-314-012 Komet ${ }^{\circledR}$ Dental, Lemgo, Germany). The bur was changed every five preparations. Two independent mesial and distal cavities were prepared in each molar. The dimensions of the cavities were standardized as follows: $4 \mathrm{~mm}$ occlusal gingival height, $4 \mathrm{~mm}$ buccolingual width and $2 \mathrm{~mm}$ axial wall depth. In the mesial cavities, the gingival margin was placed in enamel, $1 \mathrm{~mm}$ above the CEJ and in distal cavities; the gingival wall was located in cementum/ dentin, $1 \mathrm{~mm}$ below the CEJ. The teeth were kept in physiological solution until the restorative procedure. Gingival margins were left as a butt joint.

\section{Restorative procedure}

The teeth were randomly divided into four experimental groups $(n=10)$ according to restorative materials (Table 1 ).
Group 1: Dental amalgam.

Group 2: Bulk fill composite.

Group 3: Cention-N without bonding agent.

Group 4: Cention- N with bonding agent.

A Tofflemire matrix band (Shofu Dental, Mfg. Co, Ltd) was adapted firmly around the proximal aspects of the teeth. Group 1; Amalgam was condensed with hand condenser into the prepared cavities covering all walls and cavosurface margins and carved with sharp carver to the tooth contour. Polishing was done 24 hours later. Group 2; The cavities were etched with $37 \%$ phosphoric acid for 15-20s and Adper Single Bond 2 dental adhesive was applied (3M ESPE, St. Paul, MN, USA) and light cured for 20s. Next Tetric-N Ceram bulk fill composite (Ivoclar vivadent, AG, Schaan, Liechtenstein) was filled in $4 \mathrm{~mm}$ bulk increment, condensed with a condenser and was light cured for 20s. After removal of the matrix, extra curing for 20 s was performed from buccal and palatal walls.

Group 3; (self-cure mode) Cention-N powder and liquid were hand mixed to a smooth consistency according to the manufacturer's instruction. Mixing time did not exceed 60s. The cavities restored with the mixed cement in bulk and left undisturbed for $10 \mathrm{~min}$ from the beginning of mixing.

TABLE (1): Restorative materials used in the study.

\begin{tabular}{|c|c|c|}
\hline Product & Composition & Manufacturer \\
\hline Alkasite based composite (Cention -N) & $\begin{array}{l}\text { Liquid: Dimethacrylates, initiators, stabilizers, } \\
\text { additives and mint flavour. } \\
\text { Powder: Calcium fluoro-silicate glass, barium glass, } \\
\text { calcium-barium-aluminium fluoro-silicate glass, iso- } \\
\text { fillers, ytterbium trifluoride, initiators and pigments. }\end{array}$ & $\begin{array}{l}\text { Ivoclar vivadent AG } \\
\text { FL-9494 Schaan/ } \\
\text { Liechtenstein }\end{array}$ \\
\hline TetricN-Ceram BulkFill (nanohybrid) & $\begin{array}{l}\text { Dimethacrylates } 21.0 \% \text { (Bis-GMA, Bis-EMA, } \\
\text { UDMA) Polymer Filler } 17.0 \% \text { (Barium glass filler, } \\
\text { Ytterbium trifluoride) Mixed oxide } 61.0 \% \text { Additive, } \\
\text { Initiators, Stabilizers, pigments, } 1.0 \% \text {. }\end{array}$ & $\begin{array}{l}\text { Ivoclar Vivadent, } \\
\text { Schann, Liechtenstein }\end{array}$ \\
\hline $\begin{array}{l}\text { Dental amalgam } \\
\text { (vivacap amalgam) spherical } 46 \% \text { silver al- } \\
\text { loy amalgam }\end{array}$ & $\begin{array}{l}\text { Powder: Silver, tin, cupper. } \\
\text { Liquid: mercury. }\end{array}$ & $\begin{array}{l}\text { Ivoclar Vivadent Schann, } \\
\text { Liechtenstein }\end{array}$ \\
\hline
\end{tabular}


Group 4; (light cure mode) The cavities were etched and bonded like in group 2 before bulk filling with Cention-N, which was then light-cured for 20s.

Finishing and polishing for groups 2,3, and 4 was done with politip-p (NSK panaAir FX, Japan) (finishing with gray cup followed by polishing with green one).

\section{Thermal cycling and microleakage}

All the samples were stored in $37^{\circ} \mathrm{C}$ distilled water for 24 hours, before thermal cycling $(600$ thermal cycles) between $5^{\circ} \mathrm{C} / 55^{\circ} \mathrm{C}$ in water with a 30 -second dwell time ${ }^{23}$. After thermocycling, the apices of teeth were sealed with a layer of sticky wax, and all teeth surfaces were covered with two coats of fingernail polish leaving $1 \mathrm{~mm}$ around the tooth-restoration interface. The specimens then immersed in $0.5 \%$ methylene blue dye for 24 hours and thoroughly washed with distilled water, embedded in self-cured acrylic resin, and sectioned mesiodistally through the restoration's center and were examined under a stereomicroscope (Leica MZ 12; Leica Microsystems, Wetzlar, Germany) at $10 x$ magnification for dye penetration. The grading scale to assess dye penetration is outlined in Table 2. Resultant gingival microleakage scores were statistically analyzed using Kruskal Wallis followed by post-hoc Dunn's test.
TABLE (2): Microleakage Grading Scale.

\begin{tabular}{|c|l|}
\hline $\begin{array}{c}\text { Microleakage } \\
\text { Scores }\end{array}$ & \multicolumn{1}{|c|}{ Description } \\
\hline 0 & No evidence of dye penetration. \\
\hline 1 & $\begin{array}{l}\text { Dye penetration into half extension of the } \\
\text { cervical wall. }\end{array}$ \\
\hline 2 & $\begin{array}{l}\text { Dye penetration into complete extension of } \\
\text { the cervical wall. }\end{array}$ \\
\hline 3 & $\begin{array}{l}\text { Dye penetration into the cervical and axial } \\
\text { walls toward the pulp. }\end{array}$ \\
\hline
\end{tabular}

\section{RESULTS}

The results of Kruskal Wallis analysis and microleakage scores for supra CEJ and sub CEJ margins are presented in (Table3, Figure1). An intergroup comparison was done using post-hoc Dunn's test (Table 4). Microleakage was observed at all the supra CEJ and sub CEJ margins in all study groups, with statistically significant differences in groups 2 , and $4(\mathrm{p}<0.05)$ however, no significant differences were found in groups 1 and 3 ( $p>0.05)$. Significant differences in the degree of microleakage between all the materials tested $(\mathrm{P}<0.05)$ were detected with least microleakage for amalgam followed by Cention- $\mathrm{N}$ with bonding agent and Tetric-N Ceram bulk fill. And greatest leakage for Cention-N without adhesive. The difference in microleakage

TABLE (3) Microleakage scoring and comparison between leakage in supra CEJ margins and Sub CEJ margins.

\begin{tabular}{|c|c|c|c|c|c|c|c|c|c|c|}
\hline \multirow{2}{*}{\multicolumn{2}{|c|}{ No }} & \multicolumn{2}{|c|}{ Group 1} & \multicolumn{2}{|c|}{ Group 2} & \multicolumn{2}{|c|}{ Group 3} & \multicolumn{2}{|c|}{ Group 4} & $\mathrm{P}$ value \\
\hline & & $\%$ & No & $\%$ & No & $\%$ & No & $\%$ & No & \multirow{5}{*}{$<0.001 *$} \\
\hline \multirow{4}{*}{$\begin{array}{c}\text { Supra CEJ } \\
\text { margins }\end{array}$} & Score 0 & 7 & $70.0 \%$ & 5 & $50.0 \%$ & 3 & $30.0 \%$ & 4 & $40.0 \%$ & \\
\hline & Score 1 & 3 & $30.0 \%$ & 1 & $10.0 \%$ & 2 & $20.0 \%$ & 2 & $20.0 \%$ & \\
\hline & Score 2 & 0 & $0.0 \%$ & 2 & $20.0 \%$ & 1 & $10.0 \%$ & 2 & $20.0 \%$ & \\
\hline & Score 3 & 0 & $0.0 \%$ & 2 & $20.0 \%$ & 4 & $40.0 \%$ & 2 & $20.0 \%$ & \\
\hline \multirow{4}{*}{$\begin{array}{l}\text { Sub CEJ } \\
\text { margins }\end{array}$} & Score 0 & 7 & $70.0 \%$ & 2 & $20.0 \%$ & 2 & $20.0 \%$ & 2 & $20.0 \%$ & \multirow{4}{*}{$<0.001 *$} \\
\hline & Score 1 & 2 & $20.0 \%$ & 1 & $10.0 \%$ & 0 & $0.0 \%$ & 0 & $0.0 \%$ & \\
\hline & Score 2 & 1 & $10.0 \%$ & 3 & $30.0 \%$ & 4 & $40.0 \%$ & 4 & $40.0 \%$ & \\
\hline & Score 3 & 0 & $0.0 \%$ & 4 & $40.0 \%$ & 4 & $40.0 \%$ & 4 & $40.0 \%$ & \\
\hline
\end{tabular}

*: significance $<0.05$ (Kruskal Wallis analysis). 


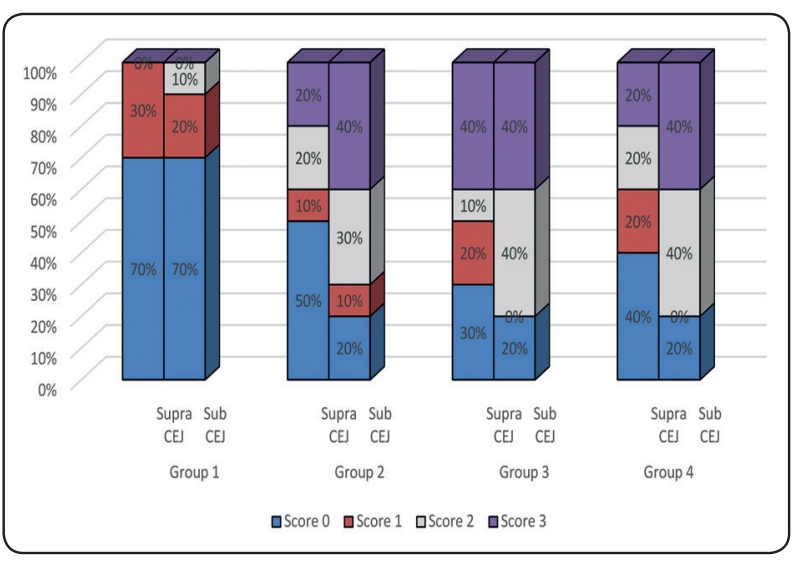

Fig. (1) Percentage of microleakage scores for the tested groups.

between Tetric-N Ceram bulk fill and Cention-N with adhesive was not significant $(\mathrm{p}>0.05)$.

TABLE (4): Post-hoc Dunn's test.

\begin{tabular}{|l|l|l|}
\hline Groups & Supra CEJ & Sub CEJ \\
\hline Group (1) / group (2) & $<0.001^{*}$ & $<0.001^{*}$ \\
\hline Group (1) / group (3) & $0.034^{*}$ & $0.03^{*}$ \\
\hline Group (2) / group (3) & $0.03^{*}$ & $0.006^{*}$ \\
\hline Group (1) / group (4) & $0.001^{*}$ & $<0.001^{*}$ \\
\hline Group (2) / group (4) & 0.84 & 0.79 \\
\hline Group (3) / group (4) & $0.019^{*}$ & $0.01^{*}$ \\
\hline
\end{tabular}

*: significance $<0.05$ (Kruskal Wallis analysis).

\section{DISCUSSION}

Class II carious lesions may extend to or below the CEJ; placing the cervical margins of restorations in dentin or cementum leading to a weaker marginal seal than at the enamel surface..$^{24}$ Microleakage test is a method of predicting the clinical behavior of the restorative materials. Different techniques are used to assess the microleakage such as radioactive isotopes, scanning electron microscope, neutron activation analysis, and dyes. Dye penetration methods are common due to low cost and simplicity of technique ${ }^{25}$. Methylene blue dye was utilized in this study due to low molecular size $(1 \mathrm{~nm})$, smaller than the diameter of dentinal tubules allowing easy penetration at tooth/restoration interface. ${ }^{26}$ Samples were exposed to thermocycling to mimic temperature changes in the oral cavity and its effect on the different restorations. 600 thermal cycles corresponding to one year of clinical service were performed $^{23}$.

According to the present study, amalgam restoration showed least microleakage which may be attributed to the precipitated corrosion products along the amalgam margins sealing the space against microleakage. The results of the present study are in agreement with Mahler et al in 2009 who compared the corrosion sealing ability of high-copper amalgams to that of low-copper amalgams and found that the sealing ability of dental amalgams is dependent on not only the kind of amalgam but also the size of the initial gap, thus there is no difference between the sealing ability of the two materials..$^{27}$

Significant difference was observed in microleakage between supra CEJ and sub CEJ margins for the bonded restorations (group 2 and 4). Dentin is rich in organic molecules, making adhesion to dentin more variable and difficult, another reason for the differences might be the good efficacy of etching and bonding to enamel, stability of enamel mineral content and limitations in the formation of proper resin tags in dentin. It is widely accepted that margins located within the dentin allow for a greater rate of microleakage than those located in the enamel. This finding is consistent with previous reports on adhesion on enamel and dentin. Greater microleakage in dentin margin of conventional and bulk fill restorations was observed by Juloski et al in 2013, which was in agreement with the results of this study. They referred the results of their studies to the decreased thickness of enamel at the gingival margin, longer distance of light curing unit from the gingival margin and weaker bond to dentin compared to enamel ${ }^{28}$. Campos et al. concluded that dentin margins show greater discontinuity than enamel margins.$^{29}$ Agarwal et al. obtained lower gingival marginal leakage in Classes II with margins in 
enamel than in dentin, concluding that the viscosity of the bulk-fill resin influences the internal adaptation in the dentine ${ }^{30}$.

Cavities restored with Cention-N with bonding agent and Tetric-N Ceram bulk-fill showed less leakage than cavities restored with Cention-N without bonding agent which may be attributed to acid etching which increases the surface area available for bonding allowing low viscosity, polymerizable resins to penetrate forming an interlocking mechanical bonding (hybrid layer)while in the cavities restored with Cention- $\mathrm{N}$ without bonding agents micromechanical interlocking was only with surface irregularities created during cavity preparation but no resin/dentin interdiffusion. No significant difference in microleakage observed with Cention- $\mathrm{N}$ with bonding agent as compared to Tetric-N Ceram bulkfill composite which may be assigned to the same bonding agent used and the isofiller technology (partially functionalized by silanes) which acts as a stress reliever keeping the shrinkage stress to a minimum. The isofiller in both materials act as a spring due to its increased elasticity (10 GP) amongst the standard glass fillers which have a higher elastic modulus of $71 \mathrm{GPa}$. High filler contents in both materials also play a role in decreasing volumetric shrinkage.

\section{CONCLUSION}

Amalgam is still the only self-sealing dental material. Cention- $\mathrm{N}$ is a new restorative material having microleakage comparable with that of packable composite, better to be used with bonding agent. More in vitro and in vivo investigations are needed to evaluate the performance of Cention- $\mathrm{N}$ because few scientific researches are available.

\section{REFERENCES}

1. Radhika M, Sajjan GS, Kumaraswamy B, Mittal NJJocdJ. Effect of different placement techniques on marginal microleakage of deep class-II cavities restored with two composite resin formulations. 2010;13(1):9.
2. de Santi Alvarenga FA, Pinelli C, Loffredo LdCMJEjod. Reliability of marginal microleakage assessment by visual and digital methods. 2015;9(01):001-05.

3. de Oliveira Carrilho MR, Tay FR, Pashley DH, Tjäderhane L, Carvalho RMJDM. Mechanical stability of resin-dentin bond components. 2005;21(3):232-41.

4. Furness A, Tadros MY, Looney SW, Rueggeberg FAJJod. Effect of bulk/incremental fill on internal gap formation of bulk-fill composites. 2014;42(4):439-49.

5. Eick J, Gwinnett A, Pashley DH, Robinson SJCRiOB, Medicine. Current concepts on adhesion to dentin. 1997; 8(3):306-35.

6. Berry TG, Nicholson J, Troendle KJTJotADA. Almost two centuries with amalgam: where are we today? 1994; 125(4):392-99.

7. Alptekin T, Ozer F, Unlu N, Cobanoglu N, Blatz MBJOd. In vivo and in vitro evaluations of microleakage around Class I amalgam and composite restorations. 2010; 35(6):641-48.

8. Burke FT, Crisp RJ, James A, et al. Two year clinical evaluation of a low-shrink resin composite material in UK general dental practices. 2011;27(7):622-30.

9. Schneider LFJ, Cavalcante LM, Silikas NJJodb. Shrinkage stresses generated during resin-composite applications: a review. 2010;2010.

10. Chen H, Manhart J, Hickel R, Kunzelmann K-HJDM. Polymerization contraction stress in light-cured packable composite resins. 2001;17(3):253-59.

11. Drummond JLJJodr. Degradation, fatigue, and failure of resin dental composite materials. 2008;87(8):710-19.

12. Aguiar F, Ajudarte KF, Lovadino JRJOd. Effect of light curing modes and filling techniques on microleakage of posterior resin composite restorations. 2002;27(6):557-62.

13. Sadeghi M, Lynch CDJOD. The effect of flowable materials on the microleakage of Class II composite restorations that extend apical to the cemento-enamel junction. 2009; 34(3):306-11.

14. Sarrett DCJDm. Clinical challenges and the relevance of materials testing for posterior composite restorations. 2005;21(1):9-20.

15. Ilie N, Bucuta S, Draenert MJOd. Bulk-fill resin-based composites: an in vitro assessment of their mechanical performance. 2013;38(6):618-25. 
16. Weinmann W, Thalacker C, Guggenberger RJDm. Siloranes in dental composites. 2005;21(1):68-74.

17. Garoushi S, Säilynoja E, Vallittu PK, Lassila LJDM. Physical properties and depth of cure of a new short fiber reinforced composite. 2013;29(8):835-41.

18. Czasch P, Ilie NJCoi. In vitro comparison of mechanical properties and degree of cure of bulk fill composites. 2013;17(1):227-35.

19. El-Damanhoury H, Platt JJOd. Polymerization shrinkage stress kinetics and related properties of bulk-fill resin composites. 2014;39(4):374-82.

20. Moorthy A, Hogg C, Dowling A, et al. Cuspal deflection and microleakage in premolar teeth restored with bulk-fill flowable resin-based composite base materials. 2012;40(6):500-05.

21. Van Ende A, De Munck J, Van Landuyt KL, et al. Bulkfilling of high C-factor posterior cavities: effect on adhesion to cavity-bottom dentin. 2013;29(3):269-77.

22. Todd JJI-VPS, Liechtenstein. Scientific Documentation: Cention N. 2016:1-58.

23. Krejci I, Lutz F, Reimer MJJoD. Marginal adaptation and fit of adhesive ceramic inlays. 1993;21(1):39-46.
24. Retief D, Mandras R, Russell CJAjod. Shear bond strength required to prevent microleakage of the dentin/restoration interface. 1994;7(1):44-46.

25. Tolidis K, Boutsiouki C, Gerasimou PJBJoOS. Microleakage in combined amalgam/composite resin restorations in MOD cavities. 2013;12:100-04.

26. Puckett AD, Fitchie JG, Bennett B, Hembree JHJQI. Microleakage and thermal properties of hybrid ionomer restoratives. 1995;26(8).

27. Mahler DB, Pham BV, Adey JDJOd. Corrosion sealing of amalgam restorations in vitro. 2009;34(3):312-20.

28. Juloski J, Carrabba M, Aragoneses JM, et al. Microleakage of Class II restorations and microtensile bond strength to dentin of low-shrinkage composites. 2013;26(5):271-7.

29. Campos EA, Ardu S, Lefever D, et al. Marginal adaptation of class II cavities restored with bulk-fill composites. 2014;42(5):575-81.

30. Agarwal RS, Hiremath H, Agarwal J, Garg AJJocdJ. Evaluation of cervical marginal and internal adaptation using newer bulk fill composites: An in vitro study. 2015;18(1):56. 